\title{
The concurrent game semantics of Probabilistic PCF
}

\author{
Simon Castellan \\ Imperial College London \\ London, United Kingdom \\ Hugo Paquet \\ University of Cambridge \\ Cambridge, United Kingdom
}

\author{
Pierre Clairambault \\ Univ Lyon, CNRS, ENS de Lyon, UCB Lyon 1, LIP \\ Lyon, France
}

\author{
Glynn Winskel \\ University of Cambridge \\ Cambridge, United Kingdom
}

\begin{abstract}
We define a new games model of Probabilistic PCF (PPCF) by enriching thin concurrent games with symmetry, recently introduced by Castellan et al, with probability. This model supports two interpretations of PPCF, one sequential and one parallel. We make the case for this model by exploiting the causal structure of probabilistic concurrent strategies. First, we show that the strategies obtained from PPCF programs have a deadlock-free interaction, and therefore deduce that there is an interpretation-preserving functor from our games to the probabilistic relational model recently proved fully abstract by Ehrhard et al. It follows that our model is intensionally fully abstract. Finally, we propose a definition of probabilistic innocence and prove a finite definability result, leading to a second (independent) proof of full abstraction.
\end{abstract}

\section{Introduction}

What is the right setting for the denotational semantics of probabilistic programs? Numerous proposals exist. Early attemps [27, 18], in the setting of domain theory, involved the probabilistic powerdomain, with which it is notoriously difficult to obtain a satisfying cartesian closed category [19]. In 2002, Danos and Harmer [12] showed that making the model more intensional offers a much more mathematically tractable development: they construct a fully abstract games model for Probabilistic Algol, an extension of Plotkin's PCF [25] with ground mutable state and probabilistic choice. Later on, Danos and Ehrhard gave a model of Probabilistic PCF (PPCF) in probabilistic coherence spaces [11], stemming from work on Linear Logic and quantitative semantics [15], and later proved to be fully abstract [14]. In a different direction, recently Staton et al [28, 16] (followed even more recently by Ehrhard et al [13]) introduced denotational models for probabilistic programming, with a focus on continuous distributions, not previously supported.

This variety of models for a large part extends existing semantics for deterministic programs. However, without probability, game semantics $[17,3]$ has offered a more modular picture, accommodating in a single framework pure fonctional computation along with computational effects such as state [4, 2], control [20], and many

Permission to make digital or hard copies of all or part of this work for personal or classroom use is granted without fee provided that copies are not made or distributed for profit or commercial advantage and that copies bear this notice and the full citation on the first page. Copyrights for components of this work owned by others than the author(s) must be honored. Abstracting with credit is permitted. To copy otherwise, or republish, to post on servers or to redistribute to lists, requires prior specific permission and/or a fee. Request permissions from permissions@acm.org.

LICS'18, Oxford, UK

(c) 2018 Copyright held by the owner/author(s). Publication rights licensed to ACM 978-1-4503-5583-4/18/07 . \$\$15.00

DOI: https://doi.org/10.1145/3209108.3209187 others ${ }^{1}$, following the well-known research programme pushed by Abramsky [1] under the name of semantic cube. Besides this modularity w.r.t. the available computational effects in the language, game semantics also offers tools to relate models. For instance, the standard cartesian closed category of Hyland-Ong games and innocent strategies embeds functorially in the relational model [6]. Under this time-forgetting operation, points of the relational model are understood as certain states reached by strategies, without any temporal information.

Of this nice picture however, little remains outside of the deterministic case. It is unclear how to equip Danos and Harmer's model [12] with a notion of probabilistic innocence extending the deterministic one, and how this model relates with alternative, less intensional semantics for probabilistic programs. In fact, even the preliminary question of non-deterministic innocence was unsolved until a few years ago $[8,30]$, when the important conceptual step was made to switch to a framework expressing explicit branching in strategies, representing more intensional behaviour. Adding quantitative information, this suggests the possibility of pushing the semantic cube towards probabilistic computation, yielding a valuable tool in our understanding of probabilistic programs.

In this paper, we make an important step in this direction. We draw on recent developments in so-called concurrent game semantics [7], a framework for game semantics built around the idea that the causality of computation (rather than plain temporal information) is primitive. In particular, we combine the thin concurrent games with symmetry $[9,10]$ of Castellan et al, used to build a parallel model of PCF [9], and the probabilistic concurrent strategies of [33]. We use this to build a games model of PPCF refining [12].

To support this model, we propose two further contributions. First, we give a quantitative extension of Boudes' theorem [6] and show that our model has a functorial collapse to the $\overline{\mathbb{R}}^{+}$-weighted relational model [21]. This builds on a key lemma independent of probabilities: that the condition of visibility from [9] ensures that composition of strategies is deadlock-free, and so inherently relational-like (an important precursor for that is Melliès' games model of Linear Logic [23]). As probabilistic coherence spaces embed faithfully in the weighted relational model, it follows by [14] that our model is intensionally fully abstract in the sense of Abramsky et al [3]. As a bonus we show that this holds both for a sequential interpretation of PPCF and for a parallel one, representing independence of sub-computations. However, definability fails.

Secondly, to get back definability we introduce a notion of sequential probabilistic innocent strategy, equivalent to standard innocent

\footnotetext{
${ }^{1}$ This significant achievement led the authors of the seminal papers on game semantics to receive last year's Alonzo Church Award for Outstanding Contributions to Logic and Computation, awarded by SIGLOG, EACSL and the Kurt Gödel Society.
} 
strategies in the deterministic case. Sequential probabilistic innocent strategies form a refined model of PPCF for which we prove finite definability (though only w.r.t. the sequential interpretation), yielding an independent proof of intensional full abstraction (in fact, unlike previously, inequational full abstraction holds).

Related work. Our probabilistic games are related to Tsukada and Ong's sheaf-based notion of innocence [29], though precise connections have not been investigated. That innocent strategies compose relationally is used in Melliès' work on game semantics for linear logic [5, 23], and exploited in Boudes' work on relating games with the relational model - our deadlock-free property generalises it to a non-sequential and non-innocent setting.

Outline. In Section 2 we introduce the semantics of probabilistic programs: we describe PPCF, its relational semantics, and the probabilistic event structures used to represent it in concurrent games. In Section 3 we develop the compositional aspects of the model, and prove the collapse to weighted relations. Finally in Section 4, we prove full abstraction: first as a consequence of the collapse, then (after adding innocence) via definability.

\section{Semantics for Probabilistic Programs}

\subsection{Probabilistic PCF}

We present the language PPCF, the extension of Plotkin's PCF [25] with a probabilistic primitive coin : Bool. Its types are those obtained from the basic types Bool and Nat, and the arrow $\Rightarrow$. Its terms are the following:

$$
\begin{aligned}
M, N::= & \lambda x \cdot M|M N| x|\mathrm{tt}| \mathrm{ff} \mid \text { if } M N_{1} N_{2} \mid Y \\
& n \mid \text { pred } M \mid \text { succ } M \mid \text { iszero } M \mid \text { coin }
\end{aligned}
$$

The typing rules are standard and omitted - we assume that in if $M N_{1} N_{2}, N_{1}$ and $N_{2}$ have ground type (Bool or Nat), a general if can be defined as syntactic sugar.

The usual call-by-name operational semantics for PCF generalises to a probabilistic reduction relation $\stackrel{p}{\rightarrow}$, for $p \in[0,1]$. All rules are straightforward, with the primitive coin representing a fair coin: coin $\rightarrow^{\frac{1}{2}} b$ for all $b \in\{\mathrm{tt}, \mathrm{ff}\}$. Because reduction is nondeterministic, there can be countably many reduction paths from $M$ to $N$, i.e. sequences of the form $M=M_{0} \stackrel{p_{1}}{\longrightarrow} \ldots \stackrel{p_{n}}{\longrightarrow} M_{n}=N$ Given such a path $\pi$, its weight $w(\pi)$ is $\prod_{1 \leq i \leq n} p_{i}$, and we define the coefficient $\operatorname{Pr}(M \rightarrow N)$ as $\sum\{w(\pi) \mid \pi$ is a path from $M$ to $N\}$.

Definition 2.1. Let $M$ and $N$ be PPCF terms such that $\Gamma \vdash M: A$ and $\Gamma \vdash N: A$. We write $M \lesssim$ ctx $N$ if for every context $C[\cdot]$ such that $\vdash C[P]:$ Bool for every $\Gamma \vdash P: A$,

$$
\operatorname{Pr}(C[M] \rightarrow b) \leq \operatorname{Pr}(C[N] \rightarrow b)
$$

for $b \in\{\mathrm{t}$, ff $\}$. The equivalence induced by this preorder, contextual equivalence, is denoted $\simeq_{c t x}$.

\subsection{The weighted relational model}

In [14], Ehrhard et al proved that probabilistic coherence spaces (PCoh) are fully abstract for PPCF: two PPCF terms are contextually equivalent iff they have the same denotation in PCoh. In fact, PCoh is cut down (via biorthogonality) from a more liberal model PRel, the $\overline{\mathbb{R}}^{+}$-weighted relational model [21], which we also refer to as the probabilistic relational model.
The relational model of PCF. Ignoring probability for now, the relational model of PCF records the input-output behaviour of a term, along with the multiplicity of resources.

Write $\mathbb{B}=\{t \mathbf{t}, \mathrm{ff}\}$ and $\mathcal{M}_{\mathrm{f}}(X)$ for the set of finite multisets of elements of a set $X$. Objects of $\mathcal{M}_{\mathrm{f}}(X)$ are written with square brackets with elements annotated with their multiplicity; e.g. we have $\left[\mathrm{tt}^{2}, \mathrm{ff}\right] \in \mathcal{M}_{\mathrm{f}}(\mathbb{B})$, where $\mathrm{tt}$ has multiplicity 2 and $\mathrm{ff}$ has multiplicity 1 . Using this notation, the term $b_{1}:$ Bool, $b_{2}:$ Bool $\vdash$ if $b_{1} b_{1} b_{2}:$ Bool will be represented as the subset of $\mathcal{M}_{\mathrm{f}}(\mathbb{B}) \times \mathcal{M}_{\mathrm{f}}(\mathbb{B}) \times \mathbb{B}$ containing:

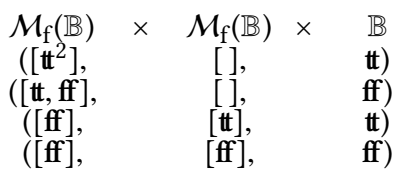

The model is non-uniform: it shows how the term behaves if its argument ever changes its mind.

The interpretation of PCF in the relational model follows the usual methodology of denotational semantics, and in particular the interpretation of the simply-typed $\lambda$-calculus in a cartesian closed category, see e.g. [22] for an introduction. To construct the target cartesian closed category, we start with one of the simplest models of linear logic: the category Rel of sets and relations. In Rel the linear logic connectives are interpreted as follows: given $X$ and $Y$, $X \otimes Y=X \multimap Y=X \times Y, X \& Y=X+Y$ (the tagged disjoint union) and $! X=\mathcal{M}_{\mathrm{f}}(X)$. The cartesian closed category Rel lis $_{\text {in }}$ then the Kleisli category for the comonad!, see e.g. [24]. We omit the details of the interpretation of PCF in Rel $_{\text {, }}$, which we will cover in the presence of probabilities.

The weighted relational model. Since the model is non-uniform, it supports non-deterministic primitives. Enriching this non-uniform model with quantitative information gives the probabilistic relational model: each element comes with a weight, as shown for instance in the interpretation of $M_{+}=b:$ Bool $\vdash$ if $b($ if coin $b \perp)$ (if $b \mathrm{ff} \mathbf{t}$ ) : Bool, where $\perp$ is a diverging term, e.g. $Y(\lambda x . x)$ :

$\begin{array}{cc}\mathcal{M}_{\mathrm{f}}(\mathbb{B}) \times & \mathbb{B}^{\frac{1}{2}} \\ \left(\left[\mathrm{tt}^{2}\right],\right. & \mathbf{t t})^{\frac{1}{2}} \\ ([\mathrm{tt}, \mathrm{ff}], & \mathrm{ff})^{\frac{3}{2}} \\ \left(\left[\mathrm{ff}^{2}\right],\right. & \mathbf{t t})^{1}\end{array}$

The weights can be greater than 1 , because a multiset may correspond to several execution traces. In the example above the pair ([tt, ff], ff) has weight $\frac{3}{2}=\frac{1}{2}+1$, summing over the different orders in which $b$ can take its values from [tt, ff].

The pure relational interpretation from before was based on the category Rel with objects sets and morphisms from $X$ to $Y$ relations $\varphi \subseteq X \times Y$, i.e. "matrices" $\left(\varphi_{x, y}\right)_{x, y \in X \times Y} \in\{0,1\}^{X \times Y}$. Accordingly, the composition of relations can be regarded as matrix multiplication: $(\psi \circ \varphi)_{x, z}=\bigvee_{y \in Y}\left(\varphi_{x, y} \wedge \psi_{y, z}\right)$.

So one may construct a probabilistic variant of Rel by simply replacing the boolean semiring $(\{0,1\}, \vee, \wedge)$ above by the semiring $\left(\overline{\mathbb{R}}_{+},+, \times\right)$where $\overline{\mathbb{R}}_{+}=\mathbb{R}_{+} \uplus\{\infty\}$ denotes the non-negative real numbers, with the infinity added to ensure convergence of the (potentially) infinite sum in the composition formula:

$$
(\psi \circ \varphi)_{x, z}=\sum_{y \in Y}\left(\varphi_{x, y} \times \psi_{y, z}\right),
$$

for $\varphi \in \mathbb{R}_{+}^{X \times Y}, \psi \in \mathbb{R}_{+}^{Y \times Z}$. 
There is a category PRel with sets as objects, and as morphisms from $X$ to $Y$ the matrices $\varphi \in \overline{\mathbb{R}}_{+}^{X \times Y}$, composed as above. The identity on $X$ is the diagonal matrix $\left(\delta_{x_{1}, x_{2}}\right)_{x_{1}, x_{2} \in X}$ where $\delta_{x_{1}, x_{2}}$ is 1 whenever $x_{1}=x_{2}$, and 0 otherwise.

Now, just like Rel, PRel supports the structure of a model of linear logic with the constructions on objects the same as in Rel and analogous constructions on morphisms. We proceed to define the interpretation of PPCF in PRel . As for Rel the interpretation of the $\lambda$-calculus combinators follows from the cartesian closed structure of the Kleisli category PRel!, which we do not detail further [22]. The interpretation of $Y$ is also obtained in a standard way as a least upper bound of finite approximations, using that homsets of PRel are dcpos when ordered componentwise. We now focus on the interpretation of ground types and associated combinators.

The types Bool and Nat are interpreted by the sets $\llbracket$ Bool $\rrbracket=$ $\mathbb{B}$ and $\llbracket \mathrm{Nat} \rrbracket=\mathbb{N}$, respectively. For $n \in \mathbb{N}$, the constant $n$ has semantics given by $(\llbracket n \rrbracket)_{k}=\delta_{k, n}$ for $k \in \mathbb{N}$. The boolean constants $\mathbf{t}$ and $\mathbf{f f}$ are interpreted in the same way. The semantics of succ and pred are defined by

$\begin{array}{lcccccc}\llbracket \text { succ } \rrbracket & : & \mathcal{M}_{\mathrm{f}}(\mathbb{N}) & \times & \mathbb{N} & \rightarrow & \overline{\mathbb{R}}_{+} \\ & ([n] & , & n+1) & \mapsto & 1 \\ & (- & , & -) & \mapsto & 0 \\ \llbracket \text { pred } \rrbracket: & \mathcal{M}_{\mathrm{f}}(\mathbb{N}) & \times & \mathbb{N} & \rightarrow & \overline{\mathbb{R}}_{+} \\ & ([n+1] & , & n) & \mapsto & 1 \\ & ([0] & , & 0) & \mapsto & 1 \\ & ([- & , & -) & \mapsto & 0\end{array}$

The morphism $\llbracket$ iszero $\rrbracket \in \operatorname{PRel}_{!}(\mathbb{N}, \mathbb{B})$ is defined similarly. Given terms $M$ : Bool, $N: \mathbb{X}, P: \mathbb{X}$ (where $\mathbb{X}$ denotes any ground type, i.e. Bool or Nat), the term if $M N P$ has semantics $\langle\llbracket M \rrbracket,\langle\llbracket N \rrbracket, \llbracket P \rrbracket\rangle\rangle \circ$ if, where if $\in \operatorname{PRel}_{!}(\mathbb{B} \&(\llbracket \mathbb{X} \rrbracket \& \llbracket \mathbb{X} \rrbracket), \llbracket \mathbb{X} \rrbracket) \cong \operatorname{PRel}(! \mathbb{B} \otimes ! \llbracket \mathbb{X} \rrbracket \otimes$ ! $\mathbb{X} \rrbracket, \llbracket \mathbb{X} \rrbracket)$ is defined by

$$
\begin{aligned}
& \text { if }: \mathcal{M}_{\mathrm{f}}(\mathbb{B}) \times \mathcal{M}_{\mathrm{f}}(\llbracket \mathbb{X} \rrbracket) \times \mathcal{M}_{\mathrm{f}}(\llbracket \mathbb{X} \rrbracket) \times \llbracket \mathbb{X} \rrbracket \rightarrow \overline{\mathbb{R}}_{+} \\
& \begin{array}{lcccccc}
([\mathrm{tt}], & {[x],} & {[]} & , & x) & \mapsto & 1 \\
([\mathrm{ff}], & {[],} & {[x]} & , & x) & \mapsto & 1
\end{array}
\end{aligned}
$$

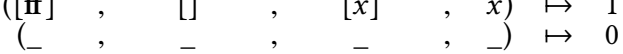

Finally, the probabilistic primitive coin is interpreted as expected as having $\llbracket \operatorname{coin} \rrbracket_{\mathbb{t}}=\frac{1}{2}$ and $\llbracket \operatorname{coin} \rrbracket_{\text {ff }}=\frac{1}{2}$, completing the interpretation of PPCF.

In order to avoid infinite weights, the authors of [14] do not stop with PRel: they cut down the category using a biorthogonality construction and obtain another weighted model of linear logic, PCoh. In PCoh weights remain finite, and the interpretation of a term of ground type $M: \mathbb{X}$ yields a sub-probability distribution on $\llbracket \mathbb{X} \rrbracket$. In fact, the main result of [14] is that PCoh is fully abstract, i.e. for any $M, N$ we have that $M \simeq_{\mathrm{ctx}} N$ iff $\llbracket M \rrbracket_{\mathrm{PCoh}}=\llbracket N \rrbracket_{\mathrm{PCoh}}$.

Interestingly this entails that, despite its drawbacks, PRel is itself already fully abstract! Indeed there is an obvious faithful forgetful functor PCoh $\hookrightarrow$ PRel preserving all the structure on the nose in fact a term $M$ has exactly the same interpretation in PRel and PCoh, the only difference being that the latter is more informative as it carries correctness information w.r.t. biorthogonality.

Although its proof is not reproduceable in PRel, the main theorem of [14] can be stated as:

Theorem 2.2. For any terms $\Gamma \vdash M: A$ and $\Gamma \vdash N:$ A of PPCF, $M \simeq_{c t x} N$ iff $\llbracket M \rrbracket_{\mathrm{PRel}}=\llbracket N \rrbracket_{\mathrm{PRel}}$.

Accordingly, in the rest of this paper, we will work only in PRel and ignore biorthogonality.

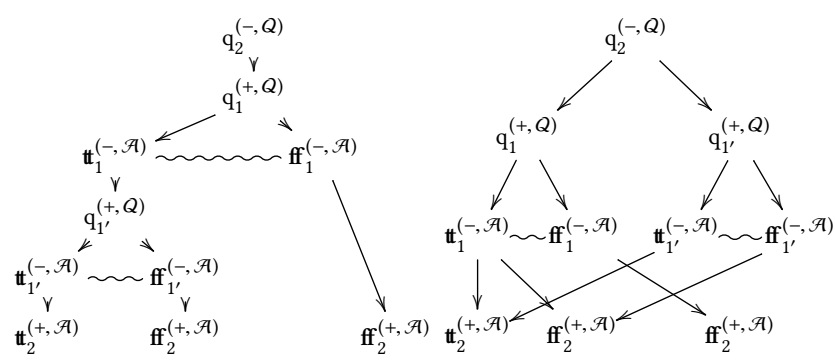

Figure 1. Two strategies for $b: \mathbf{B o o l}_{1} \vdash M=$ if $b b$ ff $: \mathbf{B o o l}_{2}$.

\subsection{Game semantics and event structures}

The interpretation of a term in PRel "flattens out" its behaviour: it only displays the multiplicity of its use of resources, but forgets in what order these resources are evaluated. This is as opposed to game semantics, which also records the order in which computational events are performed, or at least the causal dependencies between them. In the concurrent game semantics presented here (very close to [9]), the term $b:$ Bool $\vdash M=$ if $b b$ ff : Bool can be represented by either of the two diagrams in Figure 1 (i.e. there will be two interpretation functions, sending $M$ to one or the other).

These diagrams, read from top to bottom, represent dialogues (or collections of dialogues) between two players Player and Opponent, respectively playing for a program and its execution environment. Nodes, called moves, are computational events. Moves are due to either Player $(+)$ or Opponent $(-)$, as indicated by their polarity, and are annotated by a Question/Answer labelling $(Q / \mathcal{A})$ : questions correspond to variable calls, whereas answers correspond to calls returning. Wiggly lines denote incompatible branchings: moves related by them cannot occur together in an execution.

The diagram on the left is a tree, and each of its branches denotes a dialogue between Player (playing for $M$ ) and Opponent (playing for the environment) tracing one possible execution path of $M$. For instance, the leftmost path reads:

$\mathrm{q}_{2}^{(-, Q)}$ Opponent: "What is the output of $M\left(\right.$ on $\left.\mathbf{B o o l}_{2}\right)$ ?"

$\mathrm{q}_{1}^{(+, Q)}$ Player: "What is the value of $b\left(\right.$ on $\left.\mathbf{B o o l}_{1}\right)$ ?"

$\mathbf{t}_{1}^{(-, \mathcal{A})}$ Opponent: "The value of $b$ is $\mathbf{t t}$."

$\mathrm{q}_{1}^{(+, Q)}$ Player: "Then, what is, again, the value of $b$ ?"

$\mathbf{t t}_{1}^{(-, \mathcal{A})}$ Opponent: "The value of $b$ is $\mathbf{t t}$."

$\mathbf{t t}_{2}^{(+, \mathcal{A})}$ Player: "Then, the output of $M$ is tt."

In particular, this dialogue explicitly displays the several consecutive calls to $b$, leaving Opponent the opportunity to change his mind. The full diagram on the left-hand side of Figure 1 appends all such dialogues together in a single picture, the wiggly lines separating incompatible branches.

But beyond simple sequential execution, our framework for game semantics, as it is based on an independence model of concurrency, also supports a partial order-based representation of parallel executions. The diagram on the right-hand side of Figure 1 represents another implementation strategy for $M$. Taking advantage that the order of evaluation is irrelevant in PPCF, the diagram expresses that one can evaluate the two occurrences of $b$ in parallel. For each pair of results for the two independent calls to $b$, there is a Player answer to the original Opponent question $\mathrm{q}_{2}^{(-, Q)}$. Rather than just 
chronological contiguity, the arrows there describe the causal dependency of a move, i.e. the events that must have occurred before. We will see later that both diagrams denote (up to minor details, explained later) objects called strategies, representing terms. We will describe later two interpretations of PPCF as strategies: one sequential, one parallel, respectively computing the two strategies of Figure 1 from $M$.

Diagrams such as in Figure 1, that convey information about both causal dependency and incompatibility, are naturally formalised as event structures, a concurrent analogue of trees.

Definition 2.3. An event structure is $\left(E, \leq_{E}, \operatorname{Con}_{E}\right)$ with a set $E$ of events, $\leq_{E}$ a partial order stipulating causal dependency, and $\operatorname{Con}_{E}$ a non-empty set of consistent subsets of $E$, such that

$$
\begin{aligned}
& {[e]=\left\{e^{\prime} \mid e^{\prime} \leq e\right\} \text { is finite for all } e \in E} \\
& \{e\} \in \operatorname{Con}_{E} \text { for all } e \in E \\
& Y \subseteq X \in \operatorname{Con}_{E} \Longrightarrow Y \in \operatorname{Con}_{E} \\
& X \in \operatorname{Con}_{E} \text { and } e \leq e^{\prime} \in X \Longrightarrow X \cup\{e\} \in \operatorname{Con}_{E} .
\end{aligned}
$$

With an eye to game semantics, an event structure with polarity (esp) is an event structure $E$ with a function pol $: E \rightarrow\{-,+\}$.

Notations. Write $e \rightarrow e^{\prime}$ for immediate causality, i.e. $e<e^{\prime}$ with no events in between. Write $C(E)$ for the set of finite configurations of $E$, i.e. those finite $x \subseteq E$ such that $x \in$ Con and $x$ is down-closed, i.e. if $e \leq e^{\prime} \in x$ then $e \in x$. Configurations of the form $[e]$, i.e. with a top element, are called prime configurations. If $E$ has polarity, we might give information about the polarity of events by simply annotating them as in $e^{+}, e^{-}$. If $x, y \in C(E)$, write $x \subseteq^{+} y$ (resp. $x \subseteq^{-} y$ ) if $x \subseteq y$ and every event in $y \backslash x$ has positive (resp. negative) polarity.

If for an event structure $E$ there is a binary relation ${ }_{E} E$ such that for all $X \subseteq E$ finite, $X \in$ Con iff $\forall e \neq e^{\prime} \in X, \neg\left(e \#_{E} e^{\prime}\right)$, we say that $E$ has binary conflict. In that case we automatically have that if $e \# e^{\prime}$ and $e^{\prime} \leq e^{\prime \prime}$ then $e \# e^{\prime \prime}$ as well (the conflict is inherited). If $e \# e^{\prime}$ and the conflict is not inherited (meaning that for all $e_{0}<e$ and $e_{0}^{\prime}<e^{\prime}$ we have $\left.\neg\left(e_{0} \# e_{0}^{\prime}\right)\right)$, we say that $e \# e^{\prime}$ is a minimal conflict, written $e \sim e^{\prime}$. With all that in place, it should now be clear how the diagrams of Figure 1 denote event structures (with binary conflict) where rather than $\leq_{E}$ and ${ }_{E}$, we draw immediate causality $\rightarrow$ and minimal conflict $\sim$.

As strategies, we will see later that the esps of Figure 1 also come with a labelling function to a game representing the typing judgment Bool $\vdash$ Bool, labelling from which the annotations $\mathrm{q}_{2}^{(-, Q)}, \mathrm{t}_{1}^{(-, \mathcal{A})}, \ldots$ follow. But let us first discuss how probability is adjoined to event structures.

\subsection{Event structures with probability}

Sequential probabilistic esps. Sequential esps (such as that on the left of Figure 1) are those for which the causal dependency is forest-shaped, and for every configuration $x \in C(E)$, if $x$ has several distinct extensions $x \cup\left\{e_{1}^{+}\right\}, x \cup\left\{e_{2}^{+}\right\} \in C(E)$ with positive events, then $x \cup\left\{e_{1}, e_{2}\right\} \notin C(E)$. This means that for every $x \in C(E)$, there is a set of positive extensions $\operatorname{ext}_{E}^{+}(x)$, all pairwise incompatible.

Sequential esps are easily enriched with probabilities, following the game semantics of Probabilistic Idealized Algol of Danos and Harmer [12]. The basic idea is that for each $x \in C(E)$, Player equips the set of extensions $\operatorname{ext}_{E}^{+}(x)$ with a sub-probability distribution. But

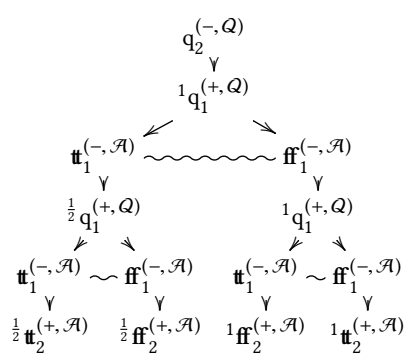

Figure 2. A probabilistic strategy for $b:$ Bool $_{1} \vdash M_{+}=$ if $b($ if coin $b \perp)($ if $b$ ff $\mathbf{t}): \mathbf{B o o l}_{2}$

rather than having a sub-distribution for each probabilistic branching in an esp, it is more convenient to carry a single valuation

$$
v: C(E) \rightarrow[0,1]
$$

putting together all the local probabilistic choices: the valuation assigned to $x$ records all the Player probabilistic choices performed in order to reach $x$. Because $v$ only records Player's probabilistic choices, it is then natural to require that (1) $v(\emptyset)=1$ and (2) $v(x \cup$ $\left.\left\{e^{-}\right\}\right)=v(x)$ for any negative extension $e^{-}$of $x$. So as to enforce that local choices give sub-probability distributions, we also have (3) for all $x \in C(E)$,

$$
v(x)-\sum_{e \in \mathrm{ext}^{+}(x)} v(x \cup\{e\}) \geq 0
$$

Furthermore, $v$ is then entirely determined by the data of $v\left(\left[e^{+}\right]\right)$ for all positive $e \in E$, hence a probabilistic sequential esp can be represented by annotating positive events with the valuation of their prime configuration. Figure 2 displays the esp to be later obtained as the interpretation of the term $M_{+}$(given in 2.2), with the probabilistic valuation written on the left of events.

General probabilistic esps. For non-sequential esps the axioms (1) and (2) still make sense, but finding the analogue of (3) is trickier, as there may be overlap between all positive extensions. This overlap leads to a redundancy in the valuation, that has to be corrected following the inclusion-exclusion principle, as in [33]:

Definition 2.4. A probabilistic esp consists in an esp $\left(E, \leq_{E}\right.$, $\operatorname{Con}_{E}, \operatorname{pol}_{E}$ ) and a valuation $v: C(E) \rightarrow[0,1]$ satisfying (1), (2) above, plus (3) if $y \subseteq^{+} x_{1}, \ldots, x_{n}$, then

$$
v(y)-\sum_{I}(-1)^{|I|+1} v\left(\bigcup_{i \in I} x_{i}\right) \geq 0
$$

where the sum ranges over $\emptyset \neq I \subseteq\{1, \ldots, n\}$ s.t. $\bigcup_{i \in I} x_{i} \in C(E)$.

We pointed out in the beginning of Section 2.3 that the deterministic term $M$ can be interpreted by either esp in Figure 1 - likewise, the probabilistic term $M_{+}$can be interpreted by the probabilistic esp of Figure 2, or by some probabilistic version of an event structure much like the right hand side diagram of Figure 1. However, unlike for sequential probabilistic esps, for general ones the valuation cannot always be pushed to events and has to remain on configurations. Consider for instance how one may assign a valuation $v$ to the esp

$$
\begin{array}{cc}
\mathrm{q}_{1}^{(-, Q)} & \mathrm{q}_{2}^{(-, Q)} \vee \\
\mathbf{t}_{1}^{(+, \mathcal{A})} & \mathbf{t}_{2}^{(+, \mathcal{A})}
\end{array}
$$


The configurations $\emptyset,\left\{\mathrm{q}_{1}\right\},\left\{\mathrm{q}_{2}\right\}$ and $\left\{\mathrm{q}_{1}, \mathrm{q}_{2}\right\}$ necessarily have coefficient 1. Consider then letting $v\left(\left\{\mathrm{q}_{1}, \mathrm{t}_{1}\right\}\right)=v\left(\left\{\mathrm{q}_{1}, \mathrm{q}_{2}, \mathrm{t}_{2}\right\}\right)=$ $\frac{1}{2}$ and $v\left(\left\{\mathrm{q}_{2}, \mathrm{t}_{2}\right\}\right)=v\left(\left\{\mathrm{q}_{1}, \mathrm{q}_{2}, \mathbf{t}_{2}\right\}\right)=\frac{1}{3}$ : nothing forces $\mathbf{t}_{1}$ and $\mathbb{t}_{2}$ to be probabilistically independent events, i.e. we may have $v\left(\left\{q_{1}, q_{2}, \mathbf{t}_{1}, \mathbf{t}_{2}\right\}\right) \neq \frac{1}{6}$. In fact the axioms would allow any value $0 \leq p \leq \frac{1}{3}$. The assignment $v\left(\left\{q_{1}, q_{2}, \mathbf{t}_{1}, \mathbf{t}_{2}\right\}\right)=\frac{1}{3}$, for example, would indicate a probabilistic dependence between $\mathbf{t}_{1}$ and $\mathbf{t}_{2}$.

\subsection{Games and strategies as esps}

So far, we have explained the formal nature of the strategies interpreting terms as (probabilistic) esps, but we have not said what games they play on.

Arenas. The games (arenas) will themselves be certain esps - a type $A$ will be interpreted by a arena $\llbracket A \rrbracket$, listing all the computational events existing in a call-by-name execution on this type and specifying the causality and compatibility constraints on these events. The arena will also remember the polarity of each event, and whether it is a question or an answer.

Consider the ground types Bool and Nat. There are only two events available between an execution environment and a term of ground type: the environment starting the evaluation of the term (Opponent question) and the evaluation finishing (Player answer). Accordingly, the corresponding arenas are:

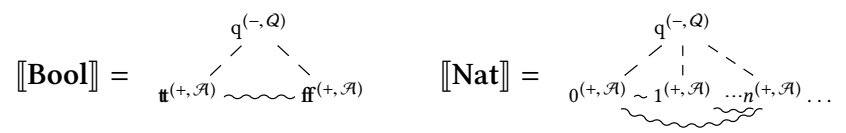

Again, the diagrams are read from top to bottom - immediate causality in arenas is represented by dashed lines rather than arrows, to keep it easily distinguishable from causality in strategies. Although the two notions have the same formal nature, they play a different role in the development.

In a typing judgment such as $\mathbf{B o o l}_{1} \vdash \mathbf{B o o l}_{2}$ there are more computational events available: upon receiving the initial question on $\mathbf{B o o l}_{2}$, Player might interrogate $\mathbf{B o o l}_{1}$, where polarity is reversed. In fact, in our running examples $M$ and $M_{+}$(from Figures 1 and 2), Player interrogates Bool $_{1}$ twice, showing the need to create copies of $\mathbf{B o o l}_{1}$. Accordingly, the sequent $\mathbf{B o o l}_{1} \vdash \mathbf{B o o l}_{2}$ will be interpreted by the arena:

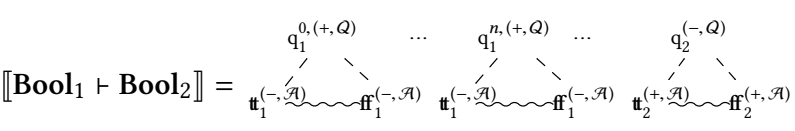

Note the new annotations $\mathrm{q}^{i,(+, Q)}$ in copies of the initial question of the argument. This copy index $i$ is implicit in the moves $\mathrm{q}_{1}^{(+, Q)}$ in Figures 1 and 2 . They will be introduced formally via an exponential modality. We now give the general definition of arenas.

Definition 2.5. An arena consists of a esp $A$, and a labelling function $\lambda_{A}: A \rightarrow\{Q, \mathcal{A}\}$ such that:

- A is a forest: if $a_{1} \leq a_{3}$ and $a_{2} \leq a_{3}, a_{1} \leq a_{2}$ or $a_{2} \leq a_{1}$.

- $A$ is alternating: if $a_{1} \rightarrow a_{2}$ then $\operatorname{pol}\left(a_{1}\right) \neq \operatorname{pol}\left(a_{2}\right)$.

- $A$ is race-free: if $a_{1} \sim a_{2}$ then $\operatorname{pol}\left(a_{1}\right)=\operatorname{pol}\left(a_{2}\right)$.

- Questions: if $a_{1}$ is minimal or if $a_{1} \rightarrow a_{2}$ then $\lambda_{A}\left(a_{1}\right)=Q$.

- Answering is affine: for every $a_{1} \in x \in C(A)$ with $\lambda_{A}\left(a_{1}\right)=$ $Q$, there is at most one $a_{2} \in x$ s.t. $a_{1} \rightarrow a_{2}$ and $\lambda_{A}\left(a_{2}\right)=\mathcal{A}$.

An arena (or esp) $A$ is negative if every minimal event is negative.
Strategies. Now that we have our notion of games, we can finish making formal the strategies displayed in Figures 1 and 2.

As pointed out earlier, the diagrams of Figure 1 have to be understood as representing esps labelled by the arena, here $\llbracket \mathrm{Bool}_{1} \vdash$ Bool $_{2} \rrbracket$. Modulo the (arbitrary) choice of copy indices for occurrences of $\mathrm{q}_{1}^{(+, Q)}$, this labelling function is implicit in the name of nodes of the diagram. However, not all such labelled esps make sense as strategies. In order to have a well-behaved notion of strategy, we will now give a number of further constraints, best introduced in multiple stages. First, we introduce pre-strategies.

Definition 2.6. A (probabilistic) pre-strategy on arena $A$ is a (probabilistic) esp $S$ along with a labelling function $\sigma: S \rightarrow A$ such that (1) for all $x \in C(S)$, the direct image $\sigma x \in C(A)$ is a configuration of the game, and (2) $\sigma$ is locally injective: for all $s_{1}, s_{2} \in x \in \mathcal{C}(S)$, if $\sigma s_{1}=\sigma s_{2}$ then $s_{1}=s_{2}$.

Conditions (1) and (2) amount to the fact that the function on events $\sigma: S \rightarrow A$ is also a map of event structures [31] from $S$ to $A$ (ignoring here the further structure on $S$ and $A$ ).

Although pre-strategies give a reasonable mathematical description of concurrent processes performed under the rules of a game (or protocol) $A$, it is too general: in particular, the current definition ignores polarity. Even in a sequential world, we expect of a definition of strategy that e.g. Player cannot constrain the behaviour of Opponent further than what is specified by the rules of the game. For our strategies on event structures, Rideau and Winskel [26] proved that we need more in order to get a category. They define:

Definition 2.7. A pre-strategy $\sigma: S \rightarrow A$ is a strategy iff it is

- receptive: for $x \in C(S)$, if $\sigma x \subseteq^{-} y \in C(A)$, there is a unique $x \subseteq x^{\prime} \in C(S)$ such that $\sigma x^{\prime}=y$; and

- courteous: for $s, s^{\prime} \in S$, if $s \rightarrow S s^{\prime}$ and if $\operatorname{pol}(s)=+$ or $\operatorname{pol}\left(s^{\prime}\right)=-$, then $\sigma s \rightarrow_{A} \sigma s^{\prime}$.

Thus a strategy can only pick the positive events it wants to play, and for each of those, which Opponent moves need to occur before. It was proved in [26] and further detailed in [7] that strategies can be composed, and form a category (up to isomorphism) whose structure we will revisit in the next section, aiming for an interpretation of PPCF.

But for now we still have some definitions to give on strategies. Indeed although at this point the causal structure of strategies is sufficiently well-behaved to fit in a compositional setting, as per usual in game semantics strategies have to be restricted further to ensure that they "behave like terms of PPCF". Typically, a set of further conditions on strategies is deemed adequate when it induces a definability result, leading to full abstraction. Here instead, our conditions will first ensure that there is a functorial collapse operation to the already fully abstract probabilistic relational model. We will add further conditions in Section 4 to prove definability.

Our conditions are a subset of those of [9]. They crucially rely on the following definition.

Definition 2.8. A grounded causal chain (gcc) in an esp $S$ is a set $\rho=\left\{\rho_{1}, \ldots, \rho_{n}\right\} \subseteq S$ such that $\rho_{1}$ is minimal in $S$ and $\rho_{1} \rightarrow S \rho_{2} \rightarrow S \rho_{3} \rightarrow S \ldots \rightarrow S \rho_{n}$. Note that some $\rho_{i}$ may have dependencies not met in $\rho$. We write $\operatorname{gcc}(S)$ for the set of gccs in $S$.

Grounded causal chains give a notion of thread in this concurrent setting. The following definition ensures that each thread can be regarded as a standalone sequential program: 


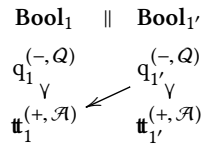

Figure 3. A non-visible strategy on Bool $_{1} \|$ Bool $_{1^{\prime}}$.

Definition 2.9. A strategy $\sigma: S \rightarrow A$ is visible iff for all $\rho \in$ $\operatorname{gcc}(S)$, we have $\sigma \rho \in C(A)$.

As arenas are forest-shaped, any non-minimal $a \in A$ has a unique predecessor just $(a) \rightarrow_{A} a$. Likewise, by local injectivity of $\sigma$, for any $s \in S$ whose image is non-minimal there is a unique $s^{\prime} \in S$, its justifier, such that $\sigma s^{\prime} \rightarrow_{A} \sigma s$, which we also write to as just(s).

With that in mind, the visibility of $\sigma: S \rightarrow A$ can be equivalently stated by asking that for all $\rho \in \operatorname{gcc}(S)$, for each $\rho_{i} \in \rho$, we have just $\left(\rho_{i}\right) \in \rho$ as well. This is reminiscent of the visibility condition in HO games, which states that the justifier of a Player move always happens within the P-view [17]. In our setting however, visibility says that a strategy can be regarded as a bag of sequential threads, sometimes forking with each other, sometimes merging, and sometimes conflicting. The strategy pictured in Figure 3 is non-visible, since the justifier of $\mathbf{t}_{1}$ is absent from the gcc $\mathrm{q}_{1^{\prime}} \rightarrow \mathbb{t}_{1}$.

Each of these sequential threads needs to respect the call-return discipline, in order to forbid strategies behaving like e.g. call/cc [20]. In a set $X \subseteq S$, we say that an answer $s_{2}^{\mathcal{A}} \in X$ (which is shortcut for $\left.\lambda_{A}\left(\sigma s_{2}\right)=\mathcal{A}\right)$ answers a question $s_{1}^{Q} \in X$ iff $\sigma s_{1} \rightarrow_{A} \sigma s_{2}$ (i.e., just $\left(s_{2}\right)=s_{1}$ ). If a $\operatorname{gcc} \rho \in \operatorname{gcc}(S)$ has some unanswered questions, we say that its pending question is the latest unanswered question, i.e. the maximal unanswered question for $\leq_{S}$.

We import from $\mathrm{HO}$ games [17]:

Definition 2.10. A visible strategy $\sigma: S \rightarrow A$ is well-bracketed iff for all $\rho=\left\{\rho_{1} \rightarrow S \ldots \rightarrow S \rho_{n+1}^{\mathcal{A}}\right\} \in \operatorname{gcc}(S), \rho_{n+1}$ answers the pending question of $\left\{\rho_{1} \rightarrow S \ldots \rightarrow S \rho_{n}\right\}$.

\section{Compositional Structure and Collapse}

\subsection{A category of games and probabilistic strategies}

We start by recalling some basic constructions on esps. Given an esp $A$, its dual is the esp $A^{\perp}$ whose events, causality and consistency are exactly those of $A$, but polarity is reversed: $\operatorname{pol}_{A^{\perp}}(a)=-\operatorname{pol}_{A}(a)$.

Given a family $\left(A_{i}\right)_{i \in I}$ of esps, we define their simple parallel composition to have events

$$
\|_{i \in I} A_{i}=\bigcup_{i \in I}\{i\} \times A_{i}
$$

with componentwise causal ordering and polarity. The consistent sets are the finite $\|_{i \in I_{0}} X_{i}$ for $I_{0} \subseteq I$ and $X_{i} \in \operatorname{Con}_{A_{i}}$ for all $i \in I_{0}$.

These constructions extend to arenas with $\lambda_{A^{\perp}}=\lambda_{A}$ and $\lambda_{\|_{i \in I} A_{i}}$ defined componentwise. A (probabilistic) strategy from $A$ to $B$ is a (probabilistic) strategy on $A^{\perp} \| B$. Sometimes we write $\sigma: A \rightarrow B$ for a strategy $\sigma: S \rightarrow A^{\perp} \| B$, keeping the $S$ anonymous.

We now show how to compose strategies. As usual in game semantics composition involves two steps: interaction and hiding. We will first show them without probabilities, and then add it back

Interaction of strategies. Let $A, B$ and $C$ be arenas, and $\sigma: S \rightarrow$ $A^{\perp} \| B$ and $\tau: T \rightarrow B^{\perp} \| C$ be strategies. Intuitively, states of the interaction $\tau \circledast \sigma$ should correspond to so-called synchronised pairs:

$$
\left\{\left(x_{S}, x_{T}\right) \mid \sigma x_{S}=x_{A}\left\|x_{B} \& \tau x_{T}=x_{B}\right\| x_{C}\right\}
$$

According to this, the interaction of $\sigma$ of Figure 3 with either $\tau_{l}$ or $\tau_{r}$ from Figure 1 (regarded as strategies on $\left(\mathbf{B o o l}_{1} \| \mathbf{B o o l}_{1^{\prime}}\right)^{\perp} \|$ Bool $_{2}$ ) would have the same maximal state

$$
\left(\left\{\mathrm{q}_{1}, \mathrm{q}_{1^{\prime}}, \mathbf{t}_{1}, \mathbf{t}_{1^{\prime}}\right\},\left\{\mathrm{q}_{2}, \mathrm{q}_{1}, \mathrm{q}_{1^{\prime}}, \mathbf{t}_{1}, \mathbf{t}_{1^{\prime}}, \mathbf{t}_{2}\right\}\right)
$$

However this seems inaccurate, because while $\sigma$ wants to play $\mathbf{t t}_{1}$ after $\mathrm{q}_{1^{\prime}}, \tau_{l}$ will only ask $\mathrm{q}_{1^{\prime}}$ after $\sigma$ plays $\mathrm{t}_{1}$ : there is a causal loop. To get an ess whose configurations correspond to causally reachable pairs of synchronised configurations, we use the following pullback in the category of esps, which we know exists from $[26,7]$ :

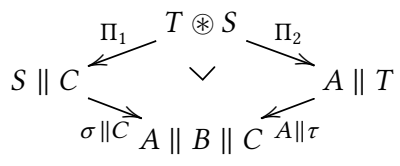

Either path around yields the interaction $\tau \circledast \sigma: T \circledast S \rightarrow A \|$ $B \| C$, a labelled event structure, charaterised in e.g. [7]:

Lemma 3.1. Configurations of $T \circledast S$ are in one-to-one correspondence with the synchronised pairs

$$
\left\{\left(x_{S}, x_{T}\right) \mid \sigma x_{S}=x_{A}\left\|x_{B} \& \tau x_{T}=x_{B}\right\| x_{C}\right\}
$$

that are causally reachable. Formally, the induced bijection $\varphi: x_{S} \|$ $x_{C} \simeq x_{A} \| x_{T}$ is secured, i.e. the relation on the graph of $\varphi$ generated by $(s, t) \triangleleft_{\varphi}\left(s^{\prime}, t^{\prime}\right)$ if $s \leq s^{\prime}$ or $t \leq t^{\prime}$ is a partial order.

In the interaction of $\sigma$ and $\tau_{l}$ above, the state $\left(\left\{\mathrm{q}_{1}\right\},\left\{\mathrm{q}_{2}, \mathrm{q}_{1}\right\}\right)$ is maximal. It cannot be extended further, as we have a deadlock: strategies are waiting on each other. This process of eliminating causal loops is the main difference between game semantics and relational semantics; and the reason why typically mapping game semantics to relational-like models is not functorial, as in e.g. [34]. Accordingly our main result will rely on Lemma 3.7, which states that the composition of visible strategies is always deadlock-free.

Composition of strategies. Following [26, 7], from $\tau \circledast \sigma: T \circledast S \rightarrow$ $A\|B\| C$, we set $T \odot S$ to comprise the events of $T \circledast S$ mapped to either $A$ or $C$, with the data of an event structure inherited. Thus, each $x \in \mathcal{C}(T \odot S)$ has a unique witness $[x]_{T \circledast S} \in \mathcal{C}(T \circledast S)$. Polarities in $T \odot S$ are set so that the restriction $\tau \odot \sigma: T \odot S \rightarrow$ $A^{\perp} \| C$ preserves them. From this we get the composition of $\sigma$ and $\tau$, a strategy $\tau \odot \sigma: T \odot S \rightarrow A^{\perp} \| C$ [7].

Composition of probabilistic strategies. We turn to the probabilistic case. For the interaction $T \circledast S$, for $x \in C(T \circledast S)$ we set:

$$
v_{T \circledast S}(x)=v_{S}\left(x_{S}\right) \times v_{T}\left(x_{T}\right)
$$

where $\Pi_{1} x=x_{S} \| x_{C}$ and $\Pi_{2} x=x_{A} \| x_{T}$. For $x \in C(T \odot S)$, we set $v_{T \odot S}(x)=v_{T \circledast S}\left([x]_{T \circledast S}\right)$. From [33], we know that this makes $\tau \odot \sigma$ a probabilistic strategy. We have defined

$$
\tau \odot \sigma: T \odot S \rightarrow A^{\perp} \| C,
$$

a probabilistic strategy from $A$ to $C$. 
The probabilistic copycat strategy. The identity strategy on an arena $A$ is the copycat strategy, $\mathfrak{c}_{A}: \mathbb{C}_{A} \rightarrow A^{\perp} \| A$. The events, consistent subsets and polarity of $\mathbb{C}_{A}$ are those of $A^{\perp} \| A$, with causality relation $\leq \mathbb{C}_{A}$ defined as the transitive closure of

$$
\begin{aligned}
\leq_{A^{\perp} \| A} \cup\left\{((1, a),(2, a)) \mid \operatorname{pol}_{A^{\perp}}(1, a)=-\right\} \\
\cup\left\{((2, a),(1, a)) \mid \operatorname{pol}_{A}(2, a)=-\right\} .
\end{aligned}
$$

Configurations of $\mathbb{C}_{A}$ are certain configurations $x_{1} \| x_{2} \in C\left(A^{\perp} \|\right.$ $A$ ). Being deterministic, copycat is easily made probabilistic by assigning probability 1 to every configuration [33]. Under these definitions the map $\propto_{A}: \mathbb{C}_{A} \rightarrow A^{\perp} \| A$ is a probabilistic strategy.

Equivalences of strategies. It is often not sensible to compare strategies up to strict equality; for instance the associativity and identity laws for composition only hold up to isomorphism of strategies. Let $\sigma: S \rightarrow A$ and $\tau: T \rightarrow A$ be probabilistic strategies on an arena $A$. A morphism from $\sigma$ to $\tau$ is a map of essp $f: S \rightarrow T$ such that $\tau \circ f=\sigma$, and for all $x \in C(S), v_{S}(x) \leq v_{T}(f x)$. Then $\sigma$ and $\tau$ are isomorphic if there are morphisms $f: S \rightarrow T$ and $g: T \rightarrow S$ of probabilistic strategies which are inverses as maps of essp.

Arenas, probabilistic strategies, and morphisms between them form a bicategory [26]. We will not use the 2-cells, so in what follows we work in the induced category (obtained by quotienting homsets). We are interested in a subcategory whose morphisms are the visible, well-bracketed strategies of Section 2.5, which are moreover negative (i.e. $S$ is negative) and well-threaded (for all $s \in S$, $[s]$ has exactly one initial move). These additional conditions are needed for the categorical structure presented in the next section.

Definition 3.2. The category PG has

- objects: negative arenas;

- morphisms from A to B: negative, well-threaded, visible and well-bracketed probabilistic strategies, up to isomorphism.

\subsection{A symmetric monoidal closed category}

Monoidal structure. The tensor $A \otimes B$ is simply defined as $A \| B$, with unit 1 the empty arena. From $\sigma_{1}: S_{1} \rightarrow A_{1}^{\perp} \| B_{1}$ and $\sigma_{2}$ $S_{2} \rightarrow A_{2}^{\perp} \| B_{2}$, form $\sigma_{1} \otimes \sigma_{2}: S_{1}\left\|S_{2} \rightarrow\left(A_{1} \otimes A_{2}\right)^{\perp}\right\|\left(B_{1} \otimes B_{2}\right)$, as obvious from $\sigma_{1} \| \sigma_{2}$; with $v_{S_{1} \otimes S_{2}}\left(x_{1} \| x_{2}\right)=v_{S_{1}}\left(x_{1}\right) \times v_{S_{2}}\left(x_{2}\right)$. Without probabilities, this yields a symmetric monoidal structure [7]; the extension with probabilities offers no difficulty.

Cartesian structure. The empty arena 1 is a terminal object. The cartesian product of arenas $A$ and $B$, written $A \& B$, has events, causality, and polarity those of $A \| B$, and consistent subsets those finite $X=X_{A} \| \emptyset$ with $X_{A} \in \operatorname{Con}_{A}$ or $X=\emptyset \| X_{B}$ with $X_{B} \in$ $\operatorname{Con}_{B}$. We have two projections:

$$
\varpi_{A}: \mathbb{C}_{A} \rightarrow(A \& B)^{\perp}\left\|A \quad \omega_{B}: \mathbb{C}_{B} \rightarrow(A \& B)^{\perp}\right\| B
$$

where one component of the \& is not reached - this is compatible with receptivity since $A$ and $B$ are negative. From $\sigma: S \rightarrow A^{\perp} \| B$ and $\tau: T \rightarrow A^{\perp} \| C$, their pairing

$$
\langle\sigma, \tau\rangle: S \& T \rightarrow A^{\perp} \|(B \& C)
$$

is obtained from $\sigma$ and $\tau$ in the obvious way. The valuation is $v_{S \& T}\left(x_{S} \| \emptyset\right)=v_{S}\left(x_{S}\right)$ and $v_{S \& T}\left(\emptyset \| x_{T}\right)=v_{T}\left(x_{T}\right)$. The incompatibility between $B$ and $C$ is key in ensuring local injectivity. Compatibility of pairing and projections, along with surjective pairing, are easy verifications.
Closed structure. Because our objects are negative arenas, $A^{\perp} \| B$ usually lies outside PG. So, inspired by the arrow construction in HO game semantics, we deviate from $A^{\perp} \| B$ by having $A$ depend on $\min (B)$ the minimal events of $B$. If there are several of them, we copy $A$ accordingly. As our setting is sensitive to linearity, we use consistency to ensure that this copying remains linear.

Definition 3.3. Consider $A, B$ two negative arenas. The arena $A \multimap B$ has as events $\left(\|_{b \in \min (B)} A^{\perp}\right) \| B$ and polarity induced. The causal order is that above, enriched with pairs $((2, b),(1,(b, a)))$ for each $b \in \min (B)$ and $a \in A$. Notice that there is a function

$$
\begin{aligned}
\chi_{A, B} \quad: \quad A \multimap B & \rightarrow A^{\perp} \| B \\
(1,(b, a)) & \mapsto(1, a) \\
(2, b) & \mapsto(2, b)
\end{aligned}
$$

collapsing all copies. We set $\operatorname{Con}_{A \multimap B}$ so as to make $\chi_{A, B}$ a map of esps, i.e. $\left(\|_{b \in \min \left(X_{B}\right)} X_{b}\right) \| X_{B} \in \operatorname{Con}_{A \multimap B}$ iff $X_{B} \in \operatorname{Con}_{B}$, $\cup_{b \in \min \left(X_{B}\right)} X_{b} \in \operatorname{Con}_{A}$, and this union is disjoint.

One may then check that there is a natural bijection $\operatorname{PG}(A \otimes$ $B, C) \cong \mathbf{P G}(A, B \multimap C)$, i.e. $\mathbf{P G}$ is symetric monoidal closed.

Dcpo-enrichment. To interpret PPCF it will be necessary for PG to be dcpo-enriched. We equip the set of probabilistic strategies on a game $A$ with a relation $\sqsubseteq$, as follows. For $\sigma: S \rightarrow A$ and $\tau: T \rightarrow A$ probabilistic strategies, set $\sigma \sqsubseteq \tau$ if $S \sqsubseteq T$, (i.e. $S \subseteq T$ and the structure of $S$ is the restriction of that of $T$ ), and if moreover $v_{S}(x) \leq v_{T}(x)$ for any $x \in C(S)$. It is clear that $\sqsubseteq$ is a partial order. The least upper bound (lub) of a directed set of probabilistic strategies is their union, with valuation given as $v(x)=\sup \left\{v_{S}(x) \mid(\sigma: S \rightarrow A) \in D\right.$ and $\left.x \in C(S)\right\}$. The least element (up to isomorphism) is given by $\perp_{A}: \min (A) \rightarrow A$ (note that the map $\emptyset \rightarrow A$ is not receptive in general and so not a strategy).

\subsection{Collapsing games and strategies}

Though we have yet to introduce a linear exponential comonad on PG to break linearity, we find it better to delay its introduction, and give now the collapse of arenas and strategies to sets and relations. Its functoriality will be addressed in the next subsection.

Mapping arenas to sets. Unlike games, PRel only records the trace of the data returned by functions for successful executions. In games, the relevant information is captured by the complete configurations, i.e. those $x$ where every question is answered in $x$.

Definition 3.4. Let $A$ be an arena. Define $\downarrow A$ to be the set of nonempty, complete configurations of $A$.

Consider for instance the arena $\llbracket$ Bool $\rrbracket$ PG for booleans. It has two nonempty and complete configurations, $\left\{q^{-}, \mathrm{tt}^{+}\right\}$and $\left\{q^{-}, \mathrm{ff}^{+}\right\}$, so $\downarrow \llbracket$ Bool $\rrbracket_{\text {PG }}$ is isomorphic to the two-element set $\{$ tt, ff $\}=\llbracket$ Bool $\rrbracket_{\text {PRel }}$.

Mapping strategies to matrices. Let $\sigma: S \rightarrow A$ be a (negative, well-threaded, visible, well-bracketed) probabilistic strategy. Our goal is to define a "vector" $\downarrow \sigma \in \overline{\mathbb{R}}_{+}^{\downarrow A}$ indexed by the nonempty and complete configurations of $A$.

Given $x \in \downarrow A$, the coefficient $(\downarrow \sigma)_{x}$ intuitively sums the probability coefficients of all the ways one can play $x$ in $S$. This is formalised using the notion of witness:

Definition 3.5. Let $\sigma: S \rightarrow A$ be a strategy and $x \in \mathcal{C}(A)$. A witness for $x$ in $\sigma$ is $z \in C(S)$ such that $\sigma z=x$, and such that all maximal moves of $z$ have positive polarity (we say $z$ is +-covered). Write $\operatorname{wit}_{S}(x)$ for the set of all witnesses of $x$ in $S$. 
The requirement that witnesses should not have negative maximal moves is illustrated by the following strategy on the game $\mathbb{B} \multimap \mathbb{B}$, where Player calls its argument and returns independently:

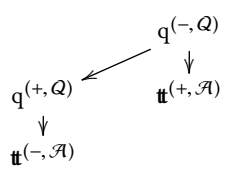

When flattening out this strategy, we must not include $(\mathbf{t}, \mathbf{t})$ as a possible execution, as this would cause functoriality to fail.

We can finally define the action of $\downarrow$ (-) on strategies.

Definition 3.6. Let $\sigma: S \rightarrow A$ be a (negative, well-threaded, visible, well-bracketed) probabilistic strategy. For $x \in \downarrow A$, we let:

$$
(\downarrow \sigma)_{x}=\sum_{z \in \text { wit }_{S}(x)} v_{S}(z) .
$$

\subsection{Functoriality of the collapse}

Following the above a morphism $\sigma: S \rightarrow A^{\perp} \| B$ in PG collapses to a vector $\downarrow \sigma$ indexed by elements of $\downarrow\left(A^{\perp} \| B\right)$. This is not quite in $\operatorname{PRel}(\downarrow A, \downarrow B)$, which would instead be indexed by elements of $\downarrow A \times \downarrow B$, i.e. pairs of nonempty configurations. For $x \| y \in \mathcal{C}\left(A^{\perp} \|\right.$ $B)$ to be nonempty it is enough for only one of $x, y$ to be nonempty. And indeed $\sigma$ might output a value without inspecting its argument: there may be witnesses to $\emptyset \| y$ in $\sigma$, so $(\downarrow \sigma)_{\emptyset \| y}$ may be non-zero. However because $A, B$ and $\sigma$ are negative, there can be no witnesses for $x \| \emptyset$ in $\sigma$, and the coefficient $(\downarrow \sigma)_{x \| \emptyset}$ is always zero.

These observations follow from PG being affine, whereas PRel is linear: a strategy can ignore its argument - and so can a morphism in the Kleisli category PRel!, but not in PRel. Thus the target of our collapse functor will not be PRel but an affine version of it introduced below. Later, moving on to the cartesian closed category $\mathrm{PG}_{\text {! }}$, we will recover the usual relational model PRel! of PPCF.

We first describe the affine version of PRel and its relationship with PRel! . After that, we prove functoriality of the collapse.

The affine relational model. Following [24, §8.10] and decompose the ! of PRel into a weakening modality ${ }_{w}^{!}$and a duplication modality ${ }_{c}^{1}$, each a comonad on PRel. For any set $X,{ }_{c}^{1} X$ contains its nonempty finite multisets: ${ }_{c}^{1} X=\mathcal{M}_{\mathrm{f}}^{\mathrm{ne}}(X)$, while ${ }_{w}^{1} X$ has the set $X$ along with the empty multiset: $\mathfrak{w}_{w} X=X+\{[]\}$. We omit details of their structure, induced from those of ! (found e.g. in [14]).

The Kleisli category PRel, is now a model of affine logic, with structure defined in terms of the structure of PRel:

- Products: the same as in PRel, $X \& Y=X+Y$.

- Monoidal structure: $X \otimes_{w} Y=X \otimes Y+X+Y$, with unit $\emptyset$.

- Closed structure: $X \multimap_{w} Y=\frac{1}{w} X \multimap Y$.

- Exponential modality: the comonad ${ }_{c}^{!}$lifted to PRel $_{i}$.

Lifting the comonad ! to PRel, $_{1}$ exploits a distributive law ${ }_{w c}^{\prime \prime} \rightarrow$ ' ' $w$, and the Kleisli category $\left(\mathbf{P R e l}_{1}\right)$, is isomorphic to PRel! . With this in place, the collapse will be a functor:

$$
\downarrow: \text { PG } \rightarrow \text { PRel }_{\downarrow}
$$

preserving the structure required for the interpretation.

We can now define the action of $\downarrow$ on a strategy $\sigma: S \rightarrow A^{\perp} \| B$ : for $x \in{ }_{w}^{\prime}(\downarrow A), y \in \downarrow B$, we set $(\downarrow \sigma)_{[], y}$ as $(\downarrow \sigma)_{\emptyset \| y}$ and $(\downarrow \sigma)_{x, y}$ as $(\downarrow \sigma)_{x \| y}$. We will now check that it is a functor, leaving the preservation of further structure for later.
A functor. Consider $\tau: T \rightarrow B^{\perp} \| C$. To show the functoriality of $\downarrow$ we must relate $\downarrow(\tau \odot \sigma)$ to the Kleisli composition $\downarrow \tau \circ \downarrow \sigma$. For $x \in{ }_{w}^{\prime} \downarrow A$ and $z \in \downarrow C$, the latter is given as:

$$
(\downarrow \tau \circ \downarrow \sigma)_{x, z}=\delta_{x,[]}(\downarrow \tau)_{[], z}+\sum_{y \in \downarrow B}(\downarrow \sigma)_{x, y}(\downarrow \tau)_{y, z},
$$

To show $\downarrow(\tau \odot \sigma)_{x, z}=(\downarrow \tau \circ \downarrow \sigma)_{x, z}$, we use a bijection between:

(1) witnesses $w$ for $x \| z$ in $\tau \odot \sigma$, and

(2) pairs $\left(w_{S}, w_{T}\right)$, where $w_{S}$ is a witness for $x \| y$ in $\sigma$, and $w_{T}$ for $y \| z$ in $\tau$, for some $y \in{ }_{w}^{\prime} \downarrow B$,

which satisfies $v_{T \odot S}(w)=v_{S}\left(w_{S}\right) \times v_{T}\left(w_{T}\right)$. There are subtleties in both directions, which we proceed to define.

From (2) to (1). This direction is the most subtle, as it bumps against the reason why traditionally operations from dynamic to static semantics are only lax functorial. Indeed, recall from Lemma 3.1 that configurations of the interaction $T \circledast S$ correspond to synchronised pairs $\left(w_{S}, w_{T}\right)$ for which the induced bijection is secured. This is in contrast with (2), where witnesses are synchronised with no securedness condition. The following crucial lemma states that, when composing visible strategies, securedness is redundant.

Lemma 3.7 (Deadlock-free lemma). Let $x_{S} \in C(S)$ and $x_{T} \in C(T)$ such that $\sigma x_{S}=x_{A} \| x_{B}$ and $\tau x_{T}=x_{B} \| x_{C}$. Then the induced bijection $\varphi: x_{S}\left\|x_{C} \simeq x_{A}\right\| x_{T}$ is secured.

So, composing visible strategies is inherently relational, from which the direction from (2) to (1) is direct.

From (1) to (2). This direction is easier: given a witness $w$ for $x \| z$ in $\tau \odot \sigma$, its down-closure $[w] \in \mathcal{C}(T \circledast S)$ satisfies $(\tau \circledast \sigma)[w]=$ $x\|y\| z$ for some $y \in C(B)$. It may look like we are done: writing $\Pi_{1}[w]=w_{S} \| z$ and $\Pi_{2}[w]=x \| w_{T}$ we obtain a pair $\left(w_{S}, w_{T}\right)$ of witnesses for $x \| y$ and $y \| z$. But it remains to check that $y \in{ }_{w}^{\prime} \downarrow B$, i.e. that it is complete. Well-bracketing ensures this.

Lemma 3.8. If $w \in$ wit $_{T \odot S}(x \| z)$, for well-bracketed visible strategies $\sigma$ and $\tau$, where $x$ and $z$ are complete, then the unique $y \in C(B)$ such that $(\tau \circledast \sigma)[w]=x\|y\| z$ is also complete.

Summing up. That this is bijective follows from +-coveredness of the witnesses; and the required equality is obtained by summing up on both sides following this bijection. The collapse preserves identities: for any arena $A, \downarrow \propto_{A}$ is the Kleisli identity $w_{w}^{\prime}(\downarrow A) \rightarrow(\downarrow A)$ (i.e. the counit for ${ }_{w}^{1}$ ). Therefore,

Theorem 3.9. $\downarrow: \mathrm{PG} \rightarrow \mathrm{PRel}_{w}$ is a functor.

Preservation of structure. This functor is well-behaved. One can easily check that it preserves the order structure on morphisms: if $\sigma \sqsubseteq \tau$ then $\downarrow \sigma \leq \downarrow \tau$, and furthermore $\downarrow\left(\bigvee_{\sigma \in D} \sigma\right)=\bigvee_{\sigma \in D}(\downarrow \sigma)$ for any directed set $D-$ so in fact $\downarrow$ (_) is itself dcpo-enriched. It behaves well also with respect to the categorical structure:

Lemma 3.10. We have the natural isomorphisms in $\mathbf{P R e l}_{1}$ :

$$
\downarrow(A \& B) \cong \downarrow A \& \downarrow B \quad \downarrow(A \| B) \cong \downarrow A \otimes_{w} \downarrow B
$$

Moreover, when $B$ has a unique initial move, we have $\downarrow(A \multimap B) \cong$ $\downarrow A \multimap w \downarrow B$. All associated structural morphisms are preserved.

\subsection{Games and strategies with symmetry}

In Section 2.5 we hinted at the need for moves to be duplicated, and adjoined copy indices. The necessity of expressing uniformity w.r.t. copy indices (see [10]) requires us to enrich our probabilistic games with a notion of symmetry. 
Probabilistic thin concurrent games. Event structures with symmetry, introduced in [32], were applied to games in [8] and refined in [9]. For lack of space we only give an informal description.

Our category is a probabilistic enrichment of the thin concurrent games of [9]. The objects are $\sim$-arenas, consisting of an arena $A$ and (among others) a set $\widetilde{A}$ of bijections $\theta: x \cong y$ between configurations $x, y \in C(A)$, expressing that $x$ and $y$ are interchangeable, i.e. the same up to copy indices. This is subject to further axioms [10], and informs an equivalence relation on $C(A)$. Likewise, probabilistic $\sim$-strategies are $\sigma: \mathcal{S} \rightarrow \mathcal{A}$ where $\mathcal{S}$ also has an isomorphism family preserved by $\sigma$, with the requirement that symmetric configurations should be assigned the same probability.

Unlike PG, this category now supports a linear exponential comonad !, whose Kleisli category is, as usual, a ccc:

Lemma 3.11. There is a cartesian closed category $\mathcal{P} \mathcal{G}_{\text {! having }}$

- objects: negative -arenas;

- morphisms $\mathcal{A}$ to $\mathcal{B}$ : (negative, well-threaded, visible, wellbracketed) probabilistic $\sim$-strategies $\sigma: \mathcal{S} \rightarrow ! \mathcal{A}^{\perp} \| \mathcal{B}$, up to isomorphism and symmetry.

Interpretation of PPCF. The interpretation of ground types as $\sim$-arenas was given in Section 2.5. It is extended to all types by setting $\llbracket A \Rightarrow B \rrbracket=! \llbracket A \rrbracket \multimap \llbracket B \rrbracket$. As a cartesian closed category, $\mathcal{P G}$ ! supports the interpretation of the simply-typed $\lambda$-calculus [22]: as usual, a typed term $\Gamma \vdash M: B$, with $\Gamma=x_{1}: A_{1}, \ldots, x_{n}: A_{n}$, is interpreted as a morphism:

$$
\llbracket M \rrbracket: !\left(\underset{1 \leq i \leq n}{\bigotimes} \llbracket A_{i} \rrbracket\right) \rightarrow \llbracket B \rrbracket
$$

It remains to interpret the primitives of PPCF. From $\Gamma \vdash M:$ Bool, $\Gamma \vdash N_{1}$ : Bool, $\Gamma \vdash N_{2}$ : Bool, we define 【if $M N_{1} N_{2} \rrbracket$ via composition with a deterministic $\sim$-strategy if: $\llbracket$ Bool $\rrbracket \& \llbracket$ Bool $\rrbracket \& \llbracket$ Bool $\rrbracket \rightarrow$

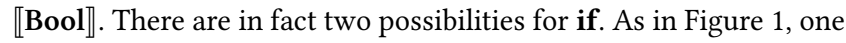
is sequential and compatible with the usual interpretation of if in game semantics, while the other is the parallel strategy from [9]. We omit the specific diagrams, hoping that they are easy to generalise from those of Figure 1. We denote the sequential and parallel interpretation by $\llbracket-\rrbracket^{s}$ and $\llbracket-\rrbracket^{p}$, respectively, and simply use $\llbracket-\rrbracket$ when the choice does not matter: in particular, both -strategies will collapse to the same weighted relation.

Finally constants are interpreted as in the following examples:

$$
\begin{aligned}
& \text { Bool } \\
& \text { Bool } \\
& \llbracket \mathrm{t} \rrbracket=\underset{\substack{\checkmark \\
\mathbf{t}^{(+, \mathcal{A})}}}{\mathrm{q}^{(-, Q)}} \\
& \llbracket \operatorname{coin} \rrbracket=
\end{aligned}
$$

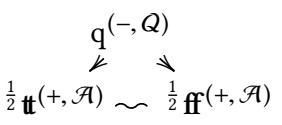

where configurations have probability 1 unless specified otherwise. For each $\sim$-arena $\mathcal{A}$, there is a (deterministic) fixpoint combinator $Y_{\mathcal{A}}$ on $(!(! \mathcal{A} \multimap \mathcal{A}))^{\perp} \| \mathcal{A}$ allowing us to interpret $Y$ as the lub of a set of approximants, see [9] for details.

Relational collapse. The new subtlety in extending our functor $\downarrow$ : PG $\rightarrow$ PRel, from Section 3.4 is that moves in ! $\mathcal{A}$ mention specific copy indices, while finite multisets $\mathcal{M}_{f}(A)$ only count multiplicity. To address that, we refine $\downarrow \mathcal{A}$ as the set of $\cong$-equivalence classes of non-empty and complete configurations of $A$ (and similarly for $\downarrow \sigma)$. The developments of Sections 3.3 and 3.4 adapt smoothly to the new framework, and we now have $\downarrow(! \mathcal{A}) \cong \mathcal{M}_{f}^{\text {ne }}(\downarrow \mathcal{A})$.
Thus, $\downarrow$ takes $\sigma: ! \mathcal{A} \rightarrow \mathcal{B}$ to $\downarrow \sigma$ in $\operatorname{PRel}_{\downarrow}(\downarrow ! \mathcal{A}, \downarrow \mathcal{B})$, which is iso to $\operatorname{PRel}(! ! ! d \mathcal{A}, \downarrow \mathcal{B}) \cong \operatorname{PRel}(! \downarrow \mathcal{A}, \downarrow \mathcal{B})$. Hence we can lift it:

Lemma 3.12. There is a functor $\downarrow: \mathcal{P G} ! \rightarrow \mathrm{PRel}_{!}$.

It is a straightforward verification that there is an isomorphism $\theta_{A}: \downarrow \llbracket A \rrbracket_{\mathrm{PG}} \cong \llbracket A \rrbracket_{\mathrm{PRel}}$ for any type $A$ of PPCF. Moreover the functor preserves the interpretation of all PPCF primitives, so that:

Theorem 3.13. For any PPCF term $\Gamma \vdash M: A$, up to the isomorphism $\theta_{\Gamma \vdash A}$, we have that $\downarrow \llbracket \Gamma \vdash M \rrbracket_{\mathcal{P} \mathcal{G}}^{s}=\downarrow \llbracket \Gamma \vdash M \rrbracket_{\mathcal{P} \mathcal{G}}^{p}=\llbracket \Gamma \vdash M \rrbracket_{\text {PRel }}$.

For instance, the probabilistic strategy for $M_{+}$from Figure 2 collapses to its relational interpretation, given in Section 2.2.

Interestingly, the equational theory on PPCF induced by the parallel interpretation is strictly finer than that induced by PRel! .

\section{Full Abstraction for PPCF}

\subsection{Full abstraction in $\mathcal{P} G$ ! by relational collapse}

We import adequacy and intensional full abstraction from PRel to $\mathcal{P} G$ ! using the functor $\downarrow$. Let $\sigma: \mathcal{S} \rightarrow \mathbb{B}$ be a probabilistic strategy. Its probability of convergence to $b \in\{\mathrm{tt}, \mathrm{ff}\}$, written $\operatorname{Pr}(\sigma \rightarrow b)$, is $\sum_{x \in C(S)} v_{S}(x)$. Applying Theorem 3.13 we get: s.t. $b \in \sigma x$

Theorem 4.1 (Adequacy). Let $\vdash M:$ Bool. Then, for $b \in \mathbb{B}$,

$$
\operatorname{Pr}(M \rightarrow b)=\operatorname{Pr}\left(\llbracket M \rrbracket \mathcal{P} \mathcal{G}_{!} \rightarrow b\right)
$$

In fact, $\mathcal{P} G$ ! is intensionally fully abstract, that is, contextual equivalence in the language coincides with contextual equivalence in the model. Let us now formally define the latter, by means of a contextual preorder. Note the similarity with Definition 2.1.

We start by defining a preorder $\leqq$ on ground type strategies: given $\sigma: S \rightarrow \mathbb{B}$ and $\tau: T \rightarrow \mathbb{B}$, write $\sigma \leqq \tau$ whenever $\operatorname{Pr}(\sigma \rightarrow$ $b) \leq \operatorname{Pr}(\tau \rightarrow b)$ for any $b \in\{\mathbf{t}$, ff $\}$. Observe that, writing $\equiv$ for the equivalence induced by $\leqq$, we have $\sigma \equiv \tau$ just in case $\downarrow \sigma=\downarrow \tau$.

Definition 4.2. If $\sigma$ and $\tau$ are probabilistic $\sim$-strategies on an arbitrary $\sim$-arena $\mathcal{A}$, write $\sigma \lesssim$ ctx $\tau$, if $\alpha \odot \Lambda(\sigma) \leqq \alpha \odot \Lambda(\tau)$ for every 'test' morphism $\alpha: \mathcal{A} \rightarrow \mathbb{B}$. The induced contextual equivalence is denoted $\simeq_{c t x}$.

Theorems 3.13 and 4.1 imply full abstraction:

Theorem 4.3 (Intensional full abstraction). Let $M$ and $N$ be PPCF terms such that $\Gamma \vdash M: A$ and $\Gamma \vdash N: A$. Then $M \simeq_{c t x} N$ if and only if $\llbracket \Gamma \vdash M \rrbracket \mathcal{P G} \simeq$ ctx $\llbracket \Gamma \vdash N \rrbracket \mathcal{P G}$ (where $\llbracket-\rrbracket$ is either $\llbracket-\rrbracket^{s}$ or $\left.\llbracket-\rrbracket^{p}\right)$.

Visible and well-bracketed probabilistic $\sim$-strategies have thus precisely the same distinguishing power as PPCF contexts. But the model still contains "junk", i.e. -strategies which do not behave like PPCF terms. In this section we impose a further condition on $\sim$-strategies (sequential innocence, defined in Section 4.2) in order to prove a finite definability result (Theorem 4.7 ). From there, a fully abstract model for PPCF follows using standard reasoning.

In what follows we simply use strategies to refer to the morphisms of $\mathcal{P} \mathcal{G}$, i.e. the negative, well-threaded, visible, and wellbracketed probabilistic $\sim$-strategies, considered up to isomorphism.

\subsection{Full abstraction by definability}

In this paper we are only concerned with definability with respect to the sequential interpretation $\llbracket-\rrbracket^{s}$ of PPCF.

Definition 4.4. A strategy $\sigma: \mathcal{S} \rightarrow \mathcal{A}$ is sequential innocent if 
- for every $x \in C(S), v(x) \neq 0$;

- a subset $X \subseteq S$ is a configuration if and only if it is an O-branching tree (that is, causality is tree-shaped and if $a \rightarrow b$ and $a \rightarrow c$ in $X$ then $\operatorname{pol}(a)=+)$ and $\sigma X \in C(A)$;

- for all $x, y, z \in C(S)$ such that $x=y \cap z$ and $y \cup z \in C(S)$,

$$
\frac{v(y \cup z)}{v(x)}=\frac{v(y)}{v(x)} \frac{v(z)}{v(x)} .
$$

The first condition is necessary for definability as configurations with probability zero are not definable in PPCF.

Sequential strategies form a well-behaved class: they are stable under composition, and copycat is sequential innocent. Call $\mathcal{P} \mathcal{G}^{\text {si }}$ the subcategory of $\mathcal{P} \mathcal{G}$ whose morphisms are (isomorphism classes of) sequential innocent strategies. We can use it to interpret PPCF:

Lemma 4.5. For any PPCF term $\Gamma \vdash M: A, \llbracket \Gamma \vdash M \rrbracket_{\mathrm{PG}}^{s}$ is a sequential innocent strategy.

So just like $\mathcal{P} \mathcal{G}_{\text {! , the category }} \mathcal{P} \mathcal{G}_{\text {! }}^{\text {si }}$ provides an adequate model of PPCF. But it is a much smaller category, allowing us to prove intensional full abstraction via definability. Fix a $\sim$-arena $\mathcal{A}=\llbracket A \rrbracket$, for $A$ some arbitrary PPCF type. As usual, finite definability will be sufficient for full abstraction:

Definition 4.6. A sequential innocent strategy $\sigma: \mathcal{S} \rightarrow \mathcal{A}$ is finite when:

- There is a bound to the length of gccs,

- For every $s^{-} \in S$, the set $\left\{t^{+} \in S \mid s \rightarrow S t\right\}$ is finite;

- $v(x) \in \mathbb{Q} \cap[0,1]$ for every $x \in C(S)$.

It is necessary for definability that configuration-valuations of finite strategies have rational coefficients (because of non-computable elements in $[0,1])$, but $\mathbb{Q}$ being dense in $\mathbb{R}$, any configurationvaluation can be approximated by ones with rational values, and indeed finite strategies form a basis for the dcpo of innocent sequential strategies on $\mathcal{A}$. Finite innocent sequential strategies have an inductive tree structure, that we exploit for PPCF definability.

Theorem 4.7 (Finite definability). For any finite $\sigma: \mathcal{S} \rightarrow \mathcal{A}$ in $\mathcal{P} \mathcal{G}_{!}^{\text {si }}$, there is a PPCF term $\vdash M:$ A such that $\llbracket M \rrbracket_{\mathcal{P} \mathcal{G}}^{s}=\sigma$.

From here, deriving a fully abstract model is standard. We write $\lesssim_{\text {ctx }}$ to denote the contextual preorder in $\mathcal{P} \mathcal{G}_{!}^{\text {si }}$ defined by requiring the $\alpha$ of Definition 4.2 to be sequential innocent. We show:

Theorem 4.8. Let $M, N$ be PPCF terms such that $\Gamma \vdash M: A$ and $\Gamma \vdash N: A$. Then, $M \lesssim_{c t x} N$ iff $\llbracket \Gamma \vdash M \rrbracket_{\mathcal{P} \mathcal{G}}^{s} \lesssim_{c t x}^{s i} \llbracket \Gamma \vdash N \rrbracket_{\mathcal{P} \mathcal{G}}^{s}$.

Note that full abstraction holds in its stronger inequational form. Definability permits this while the relational collapse did not: inequational full abstraction does not hold in PRel! [14].

\section{Conclusion}

In future work, we aim to rely on this to push further the quantitative semantic cube, studying interactions of probabilities with state and concurrency. The challenge, there, is to understand how probabilistic choice interacts with the nondeterminism of scheduling.

Acknowledgements. This work was supported by the LABEX MILYON (ANR-10-LABX-0070) of Université de Lyon, within the program "Investissements d'Avenir" (ANR-11-IDEX-0007) operated by the French National Research Agency (ANR). We also gratefully acknowledge the support of an EPSRC PhD studentship, ERC Advanced Grant ECSYM, EPSRC grants EP/K034413/1 and $\mathrm{EP} / \mathrm{K} 011715 / 1$, and the Collegium de Lyon.

\section{References}

[1] Samson Abramsky. Game Semantics for Programming Languages (Abstract). In MFCS'97, Bratislava, Slovakia, August 25-29, 1997.

[2] Samson Abramsky, Kohei Honda, and Guy McCusker. 1998. A Fully Abstract Game Semantics for General References. In Proceedings, LICS'98, Indianapolis, Indiana, USA, fune 21-24, 1998. 334-344.

[3] Samson Abramsky, Radha Jagadeesan, and Pasquale Malacaria. 2000. Full Abstraction for PCF. Inf. Comput. 163, 2 (2000), 409-470.

[4] Samson Abramsky and Guy McCusker. 1996. Linearity, Sharing and State: a fully abstract game semantics for Idealized Algol with active expressions. Electr. Notes Theor. Comput. Sci. 3 (1996), 2-14.

[5] Samson Abramsky and Paul-André Melliès. Concurrent Games and Full Completeness. In LICS'99, Trento, Italy, fuly 2-5, 1999. 431-442.

[6] Pierre Boudes. 2009. Thick Subtrees, Games and Experiments. In TLCA 2009, Brasilia, Brazil, fuly 1-3, 2009. 65-79.

[7] Simon Castellan, Pierre Clairambault, Silvain Rideau, and Glynn Winskel. 2017. Games and Strategies as Event Structures. LMCS 13, 3 (2017).

[8] Simon Castellan, Pierre Clairambault, and Glynn Winskel. Symmetry in concurrent games. In CSL-LICS '14, Vienna, 2014. 28:1-28:10.

[9] Simon Castellan, Pierre Clairambault, and Glynn Winskel. 2015. The Parallel Intensionally Fully Abstract Games Model of PCF. In Proceedings, LICS 2015, Kyoto, Japan, July 6-10, 2015. 232-243.

[10] Simon Castellan, Pierre Clairambault, and Glynn Winskel. 2016. Concurrent Hyland-Ong Games. (2016). https://arxiv.org/abs/1409.7542.

[11] Vincent Danos and Thomas Ehrhard. 2011. Probabilistic coherence spaces as a model of higher-order probabilistic computation. Inf. Comput. 209, 6 (2011), 966-991. https://doi.org/10.1016/j.ic.2011.02.001

[12] Vincent Danos and Russell Harmer. 2002. Probabilistic game semantics. ACM Trans. Comput. Log. 3, 3 (2002), 359-382.

[13] Thomas Ehrhard, Michele Pagani, and Christine Tasson. 2018. Measurable cones and stable, measurable functions: a model for probabilistic higher-order programming. PACMPL 2, POPL, 59:1-59:28.

[14] Thomas Ehrhard, Christine Tasson, and Michele Pagani. Probabilistic coherence spaces are fully abstract for probabilistic PCF. In Proceedings, POPL '14, San Diego, USA, January 20-21, 2014. 309-320.

[15] Jean-Yves Girard. 1988. Normal functors, power series and $\lambda$-calculus. Annals of pure and applied logic 37, 2 (1988), 129-177.

[16] Chris Heunen, Ohad Kammar, Sam Staton, and Hongseok Yang. 2017. A convenient category for higher-order probability theory. In LICS'17, Reykjavik. IEEE Computer Society, 1-12. https://doi.org/10.1109/LICS.2017.8005137

[17] J. M. E. Hyland and C.-H. Luke Ong. 2000. On Full Abstraction for PCF: I, II, and III. Inf. Comput. 163, 2 (2000), 285-408.

[18] Claire Jones and Gordon D. Plotkin. 1989. A Probabilistic Powerdomain of Evaluations. In (LICS '89), Pacific Grove, California, USA. IEEE Computer Society, 186-195. https://doi.org/10.1109/LICS.1989.39173

[19] Achim Jung and Regina Tix. 1998. The troublesome probabilistic powerdomain. Electr. Notes Theor. Comput. Sci. 13 (1998), 70-91.

[20] James Laird. 1997. Full Abstraction for Functional Languages with Control. In LICS'97, Warsaw, Poland, June 29 - July 2, 1997. 58-67.

[21] Jim Laird, Giulio Manzonetto, Guy McCusker, and Michele Pagani. Weighted relational models of typed lambda-calculi. In LICS'13.

[22] Joachim Lambek and Philip J Scott. 1988. Introduction to higher-order categorical logic. Vol. 7. Cambridge University Press.

[23] Paul-André Melliès. 2005. Asynchronous Games 4: A Fully Complete Model of Propositional Linear Logic. In Proceedings, LICS 2005, Chicago, IL, USA. 386-395.

[24] Paul-André Melliès. 2009. Categorical semantics of linear logic. Panoramas et syntheses 27 (2009), 15-215.

[25] Gordon D. Plotkin. 1977. LCF Considered as a Programming Language. Theor. Comput. Sci. 5, 3 (1977), 223-255.

[26] Silvain Rideau and Glynn Winskel. 2011. Concurrent Strategies. In LICS '11, fune 21-24, 2011, Toronto, Canada. 409-418.

[27] N. Saheb-Djahromi. 1980. CPO'S of Measures for Nondeterminism. Theor. Comput. Sci. 12 (1980), 19-37. https://doi.org/10.1016/0304-3975(80)90003-1

[28] Sam Staton, Hongseok Yang, Frank D. Wood, Chris Heunen, and Ohad Kammar. 2016. Semantics for probabilistic programming: higher-order functions, continuous distributions, and soft constraints. In Proceedings of LICS '16, New York, NY, USA, fuly 5-8, 2016. 525-534.

[29] Takeshi Tsukada and C.-H. Luke Ong. 2014. Innocent Strategies are Sheaves over Plays - Deterministic, Non-deterministic and Probabilistic Innocence. CoRR abs/1409.2764 (2014). http://arxiv.org/abs/1409.2764

[30] Takeshi Tsukada and C.-H. Luke Ong. 2015. Nondeterminism in Game Semantics via Sheaves. In 30th Annual ACM/IEEE Symposium on Logic in Computer Science, LICS 2015, Kyoto, Japan, fuly 6-10, 2015. 220-231.

[31] Glynn Winskel. 1986. Event Structures. In Advances in Petri Nets. 325-392.

[32] Glynn Winskel. 2007. Event Structures with Symmetry. Electr. Notes Theor. Comput. Sci. 172 (2007), 611-652.

[33] Glynn Winskel. 2013. Distributed Probabilistic and Quantum Strategies. Electr. Notes Theor. Comput. Sci. 298 (2013), 403-425.

[34] Glynn Winskel. 2013. Strategies as Profunctors. In Proceedings, FOSSACS '13, 16-24 March 2013, Rome, Italy. 418-433. 